NASA/TM- $-80-207872$

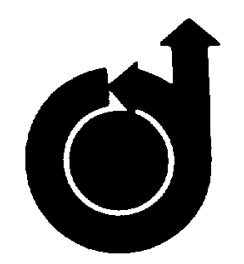

AIAA 79-0769R

Transonic Shock-Wave/Boundary-Layer

Interactions on an Oscillating Airfoil

S. S. Davis and G. N. Malcolm

$$
\begin{aligned}
& \begin{array}{l}
\text { NIS } \\
7 N-34-T M
\end{array} \\
& \begin{array}{l}
\text { NIS } \\
\text { TN-34-TM }
\end{array} \\
& 122+39 \\
& a_{1}^{2} \log _{q}^{4} 32 \\
& 1220=4
\end{aligned}
$$




\title{
Transonic Shock-Wave/Boundary-Layer Interactions on an Oscillating Airfoil
}

\author{
Sanford S. Davis* and Gerald N. Malcolm $\dagger$ \\ NASA Ames Research Center, Moffett Field, Calif.
}

\begin{abstract}
Unsteady aerodynamic londs were measured on an oscillating NACA 64A010 airfoil in the NASA Ames 11 by 11 ft Transonic Wind Tunnel. Data are presented to show the effect of the unsteady shock-wave/boundary-layer interaction on the fundamental frequency lift, moment, and pressure distributions. The data show that weak shock waves induce an unsteady pressure distribution that can be predicted quite well, while stronger shock waves cause complex frequency-dependent distributions due to flow separation. An experimental test of the principles of linearity and superposition showed that they hold for weak shock waves while flows with stronger shock waves cannot be superimposed.
\end{abstract}

\section{Nomenclature}

$C \quad=$ chord of wing, $0.5 \mathrm{~m}$

$C_{L} \quad=$ unsteady lift coefficient

$C_{L_{m}} \quad=$ static lift coefficient

$C_{L, \alpha} \quad=$ complex amplitude of unsteady lift coefficient per radian (nose up + )

$C_{M, \alpha} \quad=$ complex amplitude of unsteady moment coefficient per radian (at leading edge, nose up + )

$C_{p}=$ static pressure coefficient, $(P-P I N F) / Q I N F$

$C_{p, \alpha} \quad=$ complex amplitude of unsteady pressure coefficient per radian

$e^{i \omega t}=\cos (\omega t)+i \sin (\omega t)$

$f=$ frequency, $\mathrm{Hz}$

$k \quad=$ reduced frequency, $\omega C / 2 U$

$M \quad=$ freestream Mach number

$P \quad=$ local static pressure, $\mathrm{N} / \mathrm{m}^{2}$

$P I N F=$ freestream static pressure, $\mathrm{N} / \mathrm{m}^{2}$

QINF = freestream dynamic pressure, $\mathrm{N} / \mathrm{m}^{2}$

$\operatorname{Re} \quad=$ chord Reynolds number

$U=$ freestream velocity, $\mathrm{m} / \mathrm{s}$

$\alpha \quad=$ complex amplitude of unsteady angle of attack

$\alpha_{m} \quad=$ static angle of attack

$\omega \quad=$ radian frequency, $\omega=2 \pi f$

\section{Introduction}

$\mathbf{U}$ NSTEADY flows are one of the least understood problem areas in fluid mechanics. This is particularly true in the critical transonic flow regime where nonlinear and dissipative effects tend to dominate the flow. Although recent advances in computational aerodynamics have made it possible to compute unsteady inviscid flows, unsteady flows with strong viscous interactions are not adequately modeled. Because of this lack of a computational model, the present understanding of transonic unsteady flows has been developed mostly through experimental studies.

Transonic unsteady airfoil testing was initially motivated by the destructive ef fects of aileron buzz encountered on highspeed aircraft during World War II. Early work at the (then) NACA Ames Aeronautical Laboratory by Erickson and his

Presented as Paper 79-0769 at the AIAA/ASME/ASCE/AHS 20th Structures, Structural Dynamics, and Materials Conference. St Louis, Mo., April 4-6, 1979; submitted May 4, 1979; revision received Jan. 29, 1980. This paper is declared a work of the U.S. Government and therefore is in the public domain.

Index categories: Nonsteady Aerodynamics; Transonic Flow; Aeroelasticity and Hydroelasticity.

- Research Scientist. Associate Fellow AIAA.

†Research Scientist. Member AIAA. coworkers ${ }^{1.2}$ on control surface oscillations culminated in the development and operation of a large test rig utilizing splitter plates in the (then) Ames 16-ft High-Speed Wind Tunnel. Unsteady loads on oscillating airfoils and control surfaces were measured in a series of tests over a five-year period. ${ }^{3-7}$ This early work at Ames was instrumental in developing miniature instrumentation and refining the newly acquired unsteady testing capability. Parallel work at the (then) NACA Langley Aeronautical Laboratory ${ }^{8-10}$ clarified the effect of unsteady wall interference and wind-tunnel resonance. A summary of British and European work on oscillating airfoils in this period may be found in Refs. 11 and 12. All this early unsteady research was hampered at supercritical speeds by choking of the older unventilated wind tunnels.

After almost 20 years of inactivity in two-dimensional transonic unsteady aerodynamics, there is currently an urgent technological need for a more complete understanding of these flows. The work of Tijdeman and his co-workers at the National Aerospace Laboratory (NLR), Amsterdam, The Netherlands, has motivated much of the current research. Using a carefully calibrated pneumatic-tubing system, they were able to extract first-harmonic unsteady aerodynamic pressures on models ranging from oscillating flaps to supercritical airfoils. ${ }^{13-15}$ Many of the new physical phenomena observed and reported in Refs. 13-15 are described in detail in Ref. 15. Recently, many other research groups have initiated oscillating airfoil experiments. Results of these investigations, mostly on smaller models, may be found in the proceedings of recent national and international conferences. ${ }^{16,17}$ The current experimental investigations at Ames were designed to expand the existing unsteady aerodynamic test envelope to higher Reynolds numbers, to different modes of motion, and to more diverse mean flow conditions.

The Ames oscillating airfoil experiment was run in the 11 by $11 \mathrm{ft}$ transonic wind tunnel. A $0.5 \mathrm{~m}$ chord $\times 1.35 \mathrm{~m}$ span wing with an NACA 64A010 airfoil section was installed in the test section between two splitter plates as shown in Fig. 1. The model was driven into a pitching or plunging motion by a servocontrolled hydraulic actuation system. Motion was imparted to the wing via four push-pull rods imbedded in the splitter plates. The unsteady data were measured with an array of miniature in-situ piezoresistive pressure transducers. Steady data were measured with conventional pneumatic instrumentation. All the data were input directly into a minicomputer-based data system for subsequent online data reduction and analysis. More detailed descriptions of the test apparatus, wing model, instrumentation, and computer system may be found in Refs. 18-20. 


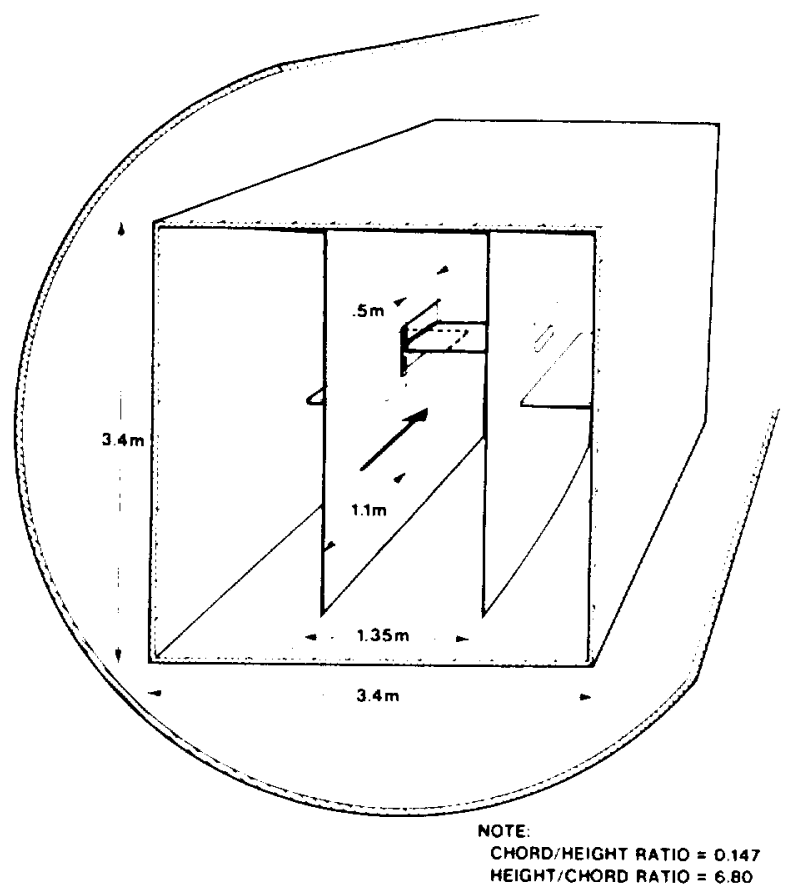

Fig. 1 Oscillating airfoil installed in Ames 11 by 11 fl Transonic Wind Tunnel.

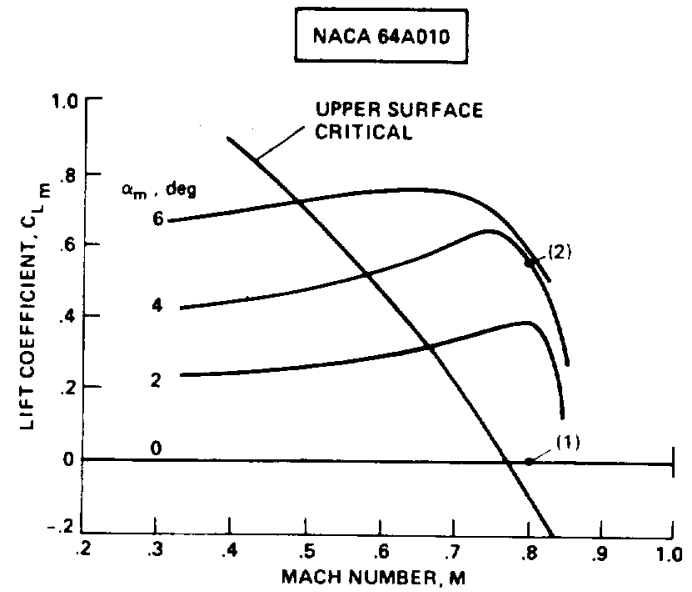

Fig. 2 Measured static-response curves for NACA 64 A010 airfoil.

The purpose of this paper is to discuss selected portions of the data that illustrate the complicated aerodynamic effects on as oscillating airfoil at transonic speeds. An especially important component-one that is stressed in this paper-is the effect of the shock-wave/boundary-layer interaction on the unsteady loads. This effect is illustrated by examining in detail the first-harmonic unsteady pressure distributions at the two mean flow conditions shown in Fig. 2. Condition 1 represents a transonic flow with a weak shock wave, while condition 2 is an example of a transonic flow with shockinduced separation (shock stall). For each of these conditions first-harmonic unsteady pressure distributions, generalized forces (lift and moment), and selected comparisons with theories are presented. These comparisons will show that weak shock waves induce an unsteady aerodynamic response that is amenable to present-day computational techniques. It is also shown that under shock stall conditions the unsteady pressures are not well predicted by an inviscid analysis and strong frequency-dependent viscous effects appear. The paper concludes with an experimental verification of the principles of linearity and superposition for these two conditions. These principles are routinely assumed in present-day practice and

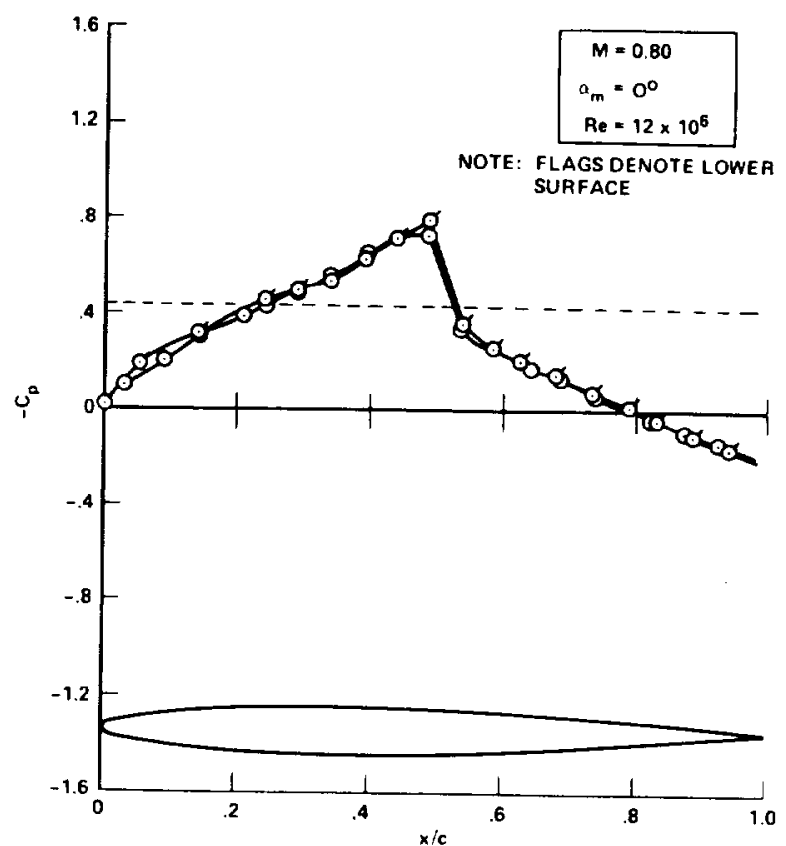

Fig. 3 Static pressure distribution in transonic flow (condition 1).

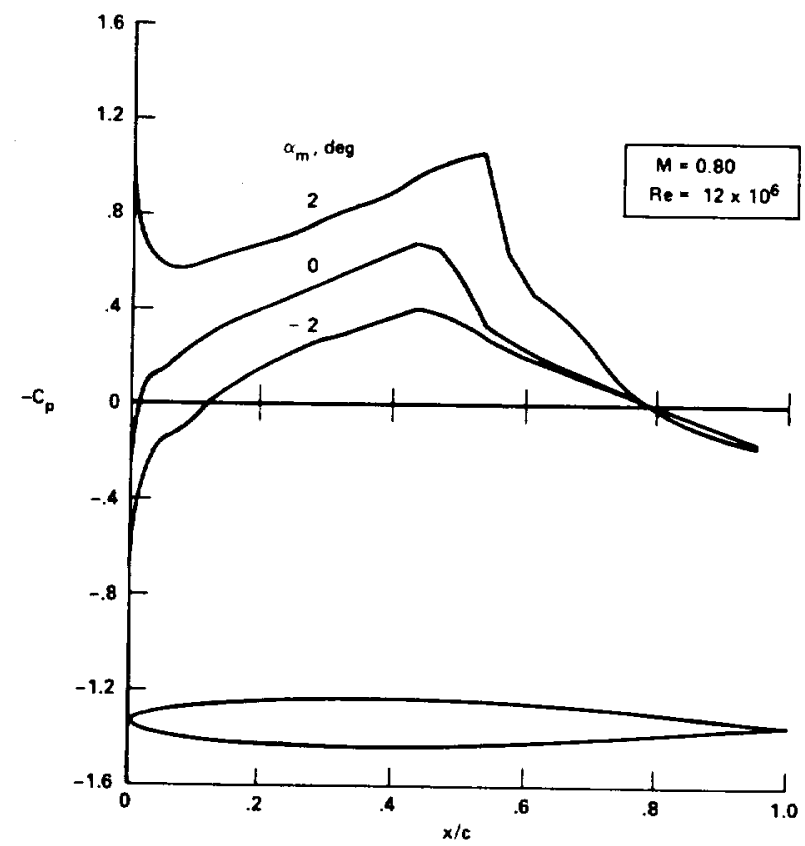

Fig. 4 Effect of mean angle on static pressure distribution in transonic flow (condition 1).

their limits of validity must be fully explored before meaningful transonic analyses can proceed.

The data presented in this paper form a small part of the extensive data bank that has been assembled from this experiment. Some other data may be found in Ref. 19 and a first-harmonic analysis of the complete data set may be found in Ref. 21. Detailed information on the geometry of the NACA 64A010 test airfoil may be found in Refs. 22 and 23.

\section{Transonic Unseparated Flow}

This case is represented by condition 1 in Fig. 2 and lies just to the right of the boundary separating subcritical and supercritical flows. The supercritical flow region is not extensive, and it is terminated by a weak normal shock. The flow is expected to be attached over the entire airfoil and, as will be shown, inviscid-flow modeling adequately predicts the 
PITCHING 1: $0.25 C$
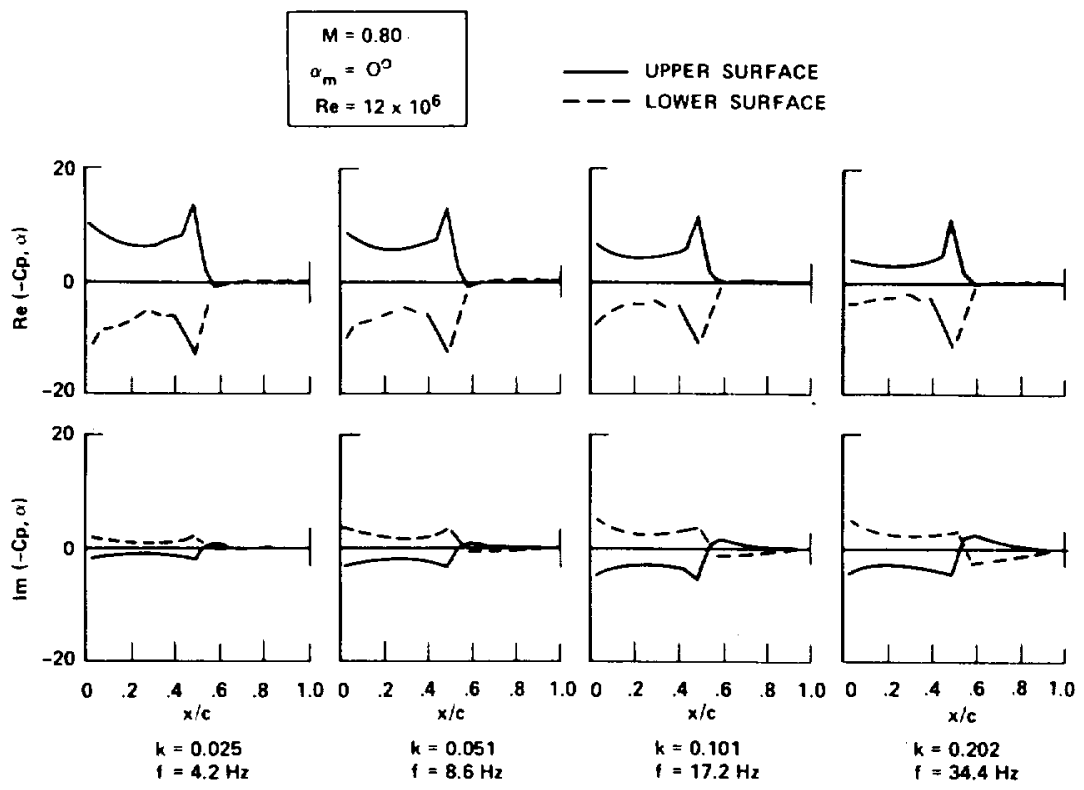

Fig. 5 Effect of frequency on unsteady pressure distributions.

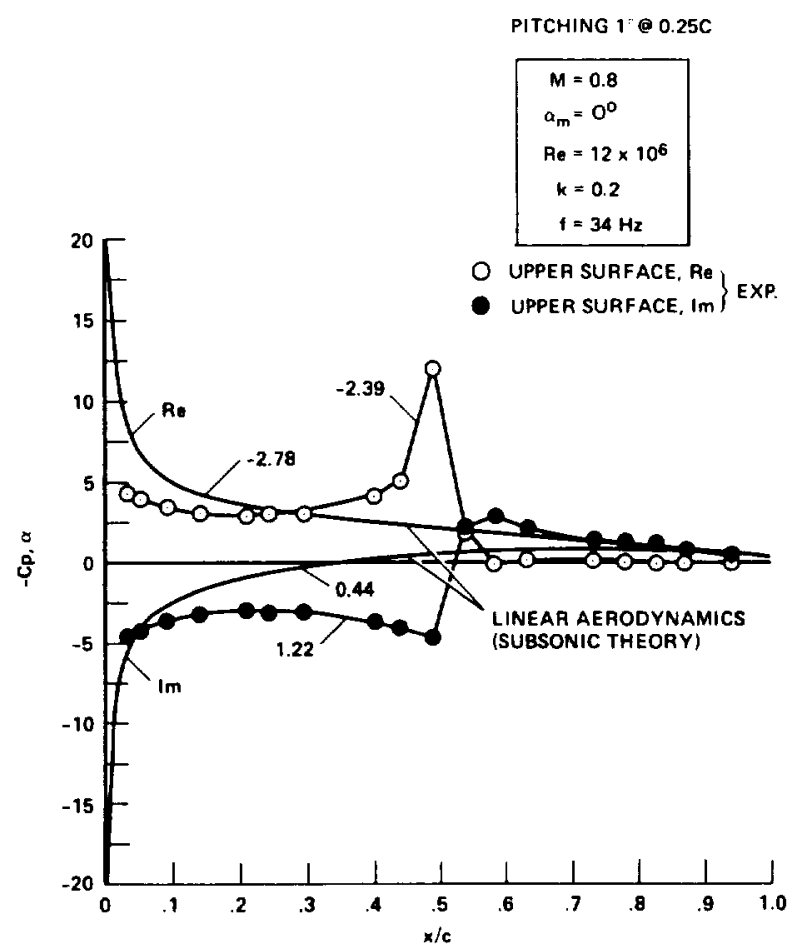

Fig. 6 Upper-surface unsteady pressure distribution compared with subsonic theory.

major features of the unsteady pressure distribution. The static pressure distribution is shown in Fig. 3, where a weak shock wave is shown at approximately midchord. Downstream of the shock wave the mild adverse pressure gradient insures good pressure recovery to the trailing edge.

The quasisteady response for the pitching mode is shown in Fig. 4. Since the motion is symmetric, only upper-surface pressures are shown. The magnitude of the unsteady pressure is approximately proportional to the distance between the $\alpha_{m}= \pm 2$ deg curves at each $X / C$ station. Starting from the trailing edge, the response is minimal over the latter $25 \%$ chord and increases rapidly in the vicinity of the shock wave. Upstream of the shock, the response is uniformly large. This behavior differs from that found in both subcritical experiments and linear theory where the unsteady amplitude

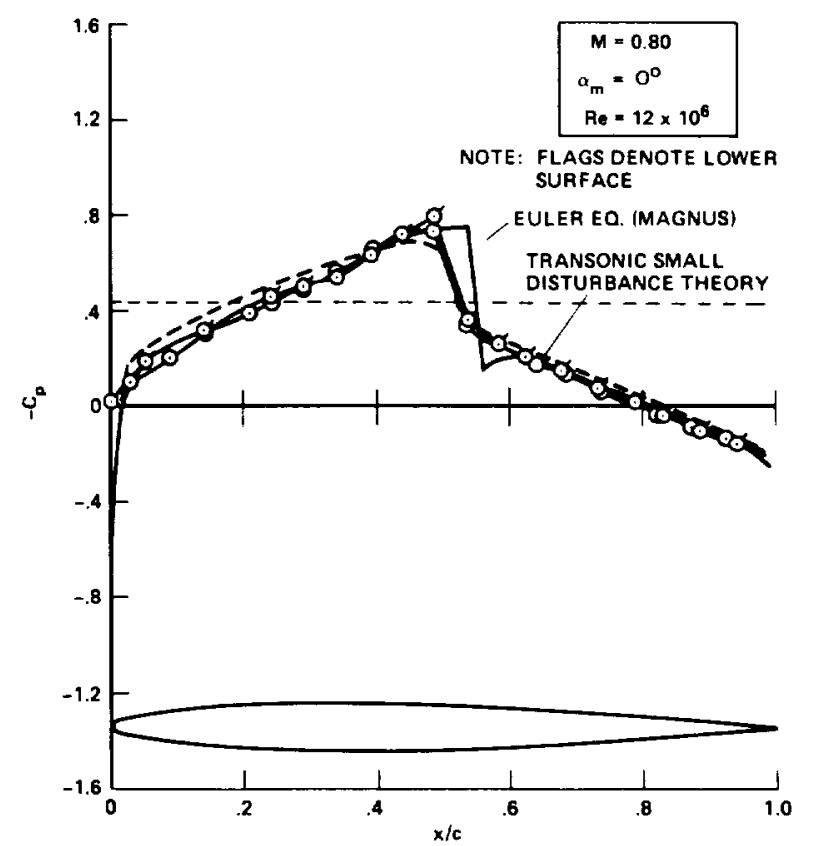

Fig. 7 Static pressure distribution compared with numerical solutions.

increases smoothly from the trailing edge. ${ }^{19}$ As will be shown, this flow condition is representative of Tijdeman's Type II-I motion (Ref. 15, p. 72) since the weak shock wave disappeared during a portion of the cycle. The shock-wave movement (where it exists) is consistent with inviscid-flow theory since it moves aft with increasing angle of attack.

The measured unsteady pressure at the fundamental frequency is shown in Fig. 5 for a pitching mode. The real part is consistent with the quasisteady analysis given previously. The unsteady pressure is very small in the trailingedge region, increases rapidly to a peak at the shock wave, and remains fairly level over the forward half of the airfoil. Its shape remains constant over the entire frequency range, but the amplitude decreases slightly with frequency. The imaginary part is out of phase with the motion upstream of the shock, but it quickly undergoes a 180 deg phase change at the shock. Unlike the real component, the imaginary component is locally antisymmetric with respect to the shock 
position. Starting from a low level, the imaginary component increases with frequency to a level approaching that of the real component. The imaginary components have both positive and negative contributions to the loads. Also, unlike the real component, the imaginary component contributes significant postshock pressure disturbances. The unsteady pressures on the lower surface of this symmetric airfoil are always $180 \mathrm{deg}$ out of phase with those on the upper surface.

A comparison of the supercritical unsteady pressure distribution with linear subsonic theory at $k=0.20(f=34 \mathrm{~Hz})$ is shown in Fig. 6. The effect of the shock wave clearly invalidates the linear theory. Both the real and imaginary parts predicted by the theory are wrong. However, some interesting integrated results were obtained by using linear subsonic theory. When the areas under the real components were computed they were unexpectedly close. The real component of the lift and the center-of-pressure position are actually predicted quite well by using linear theory. (Notice that the "center-of-gravity" of the experimental curve is at $25 \%$ chord since the loading drops to zero downstream of the shock.) This result must be considered fortuitous and is probably accounted for by the favorable shock-wave position. The imaginary part is not well predicted, and even the real part must be quite sensitive to shock-wave position.

A calculation was made by Magnus ${ }^{24}$ for this flow condition by using the Euler equations of motion. Magnus' mean flow computation is shown in Fig. 7 along with the prediction based upon a steady transonic small-disturbance code ${ }^{25}$ (program TSFOIL). The predictions based upon Euler's equation show good agreement with the data except for a slightly downstream shock-wave position. The smalldisturbance theory predicts a slight overexpansion over the forward part of the airfoil relative to both the experiment and Euler's equation. The adverse pressure gradients approaching the trailing edge are predicted quite well, an indication of fully attached flow over the entire airfoil.

An unsteady pressure distribution from a harmonic analysis of Magnus' data is shown in Fig. 8. The experimental data are the same as those presented in Fig. 6. Upstream of the shock wave, both the real and imaginary components are predicted quite well. The theoretical unsteady pressures caused by the

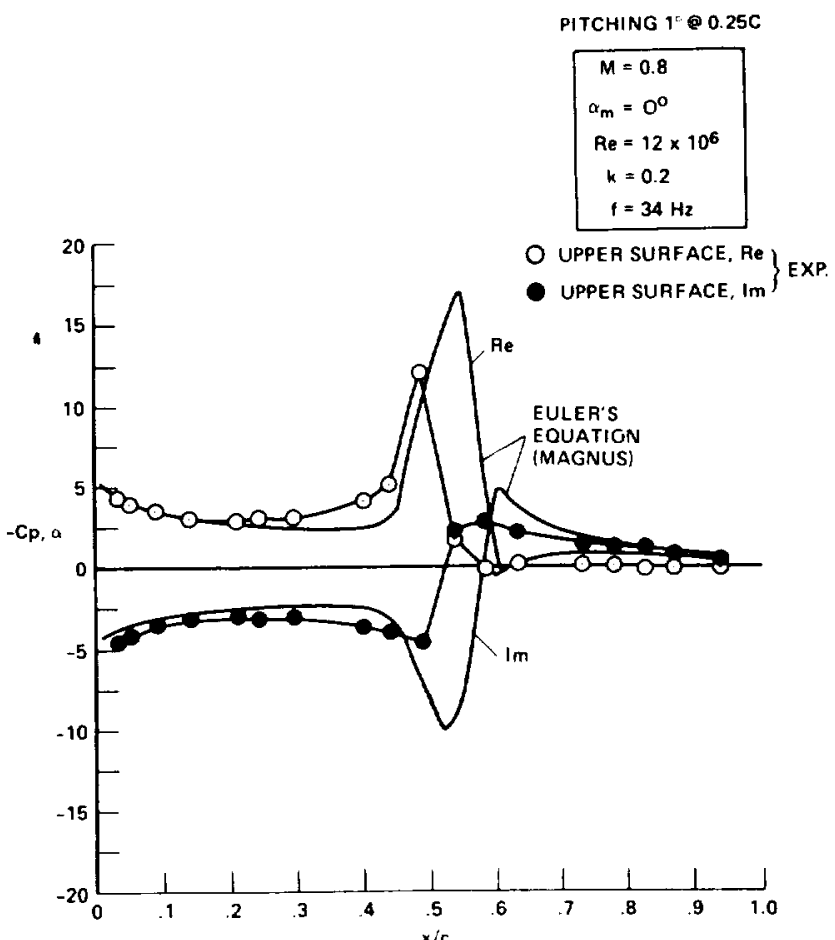

Fig. 8 Upper-surface unsteady pressure distribution compared with numerical solution of Euler's equation. shock motion are overpredicted, and the postshock response is also overpredicted. This behavior is to be expected because the forward region of the airfoil is influenced mainly by the inviscid flow, while the shock dynamics and, to some extent, the postshock behavior must be influenced by the unsteady shock-wave/boundary-layer interaction. More extensive comparisons of these data with another solution of Euler's equation that includes some viscous effects may be found in Ref. 26. Overall this result is very encouraging, and the outlook is that more efficient codes that include viscous effects will be able to predict these flows quite well.

The aerodynamic transfer function for this flow condition is shown in Fig. 9. The unsteady lift and moment is similar in many ways to that predicted by linear theory even though the flow is supercritical. The magnitude (fortuitously) agrees quite well with linear subsonic theory, but the phase lag of the supercritical data with respect to subsonic theory is quite large. This is caused by a combination of wake disturbances and some contributions from the shock wave. Scale effects do not seem to be important for this flow condition. For the reasons discussed previously, the predictions based upon the Euler equation overestimate the loads.

\section{Transonic Flows with Shock-Induced Separation}

The flow condition indicated by point 2 in Fig. 2 is representative of a transonic flow with a strong shock wave and subsequent flow separation. As will be shown, the oscillatory pressures exhibit many new characteristics that are not modeled by inviscid flow theory. The static pressure distribution is presented in Fig. 10. The shock wave is stronger and farther upstream than that shown in Fig. 3. The poor trailing-edge pressure recovery indicates probable flow separation. Interferograms obtained by Johnson and Bachalo ${ }^{27}$ on a $15 \mathrm{~cm}$ chord model of the same airfoil clearly

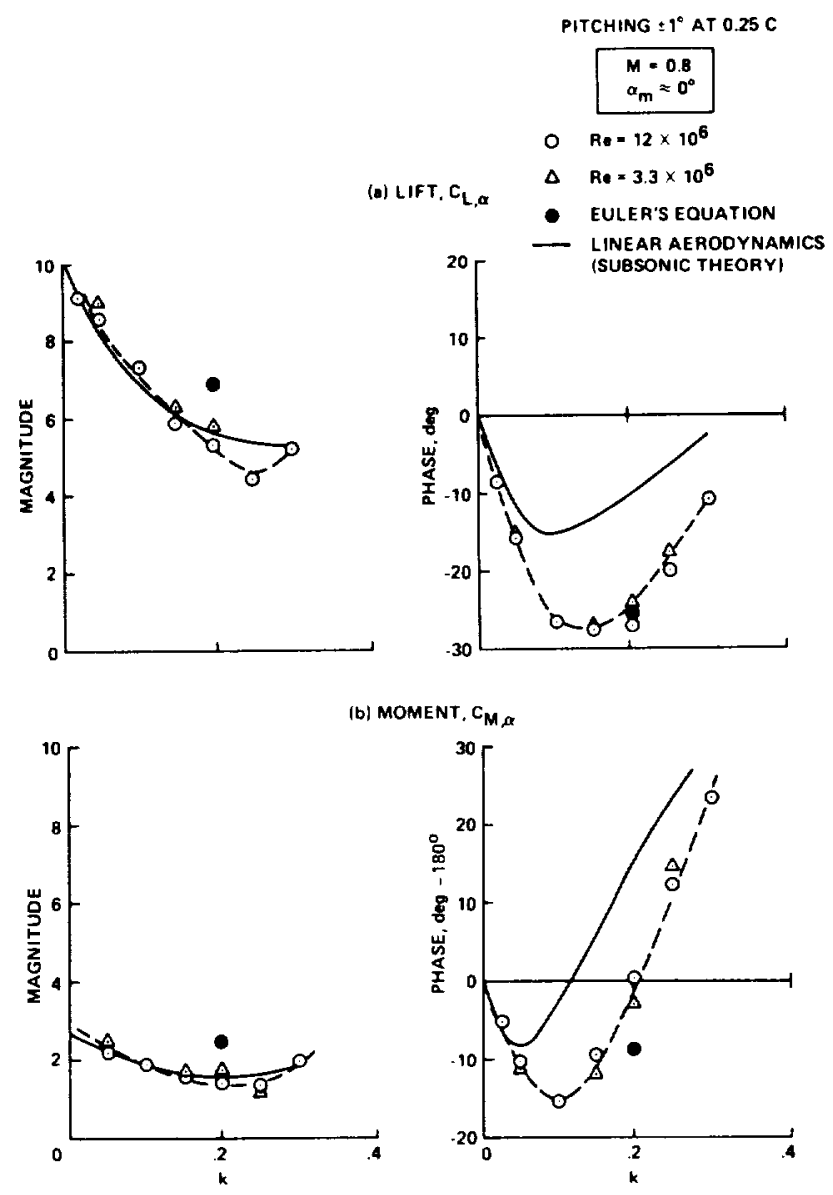

Fig. 9 Aerodynamic transfer function for transonic flow: a) lift coefficient, b) leading-edge moment coefficient (both per radian). 


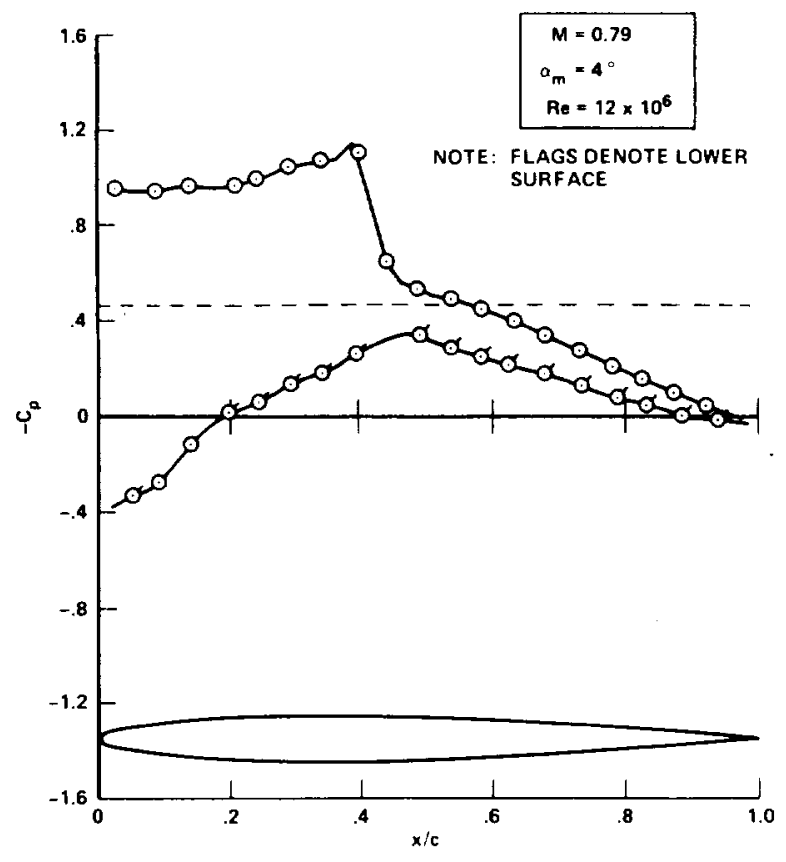

Fig. 10 Static pressure distribution for shock stall (condition 2).

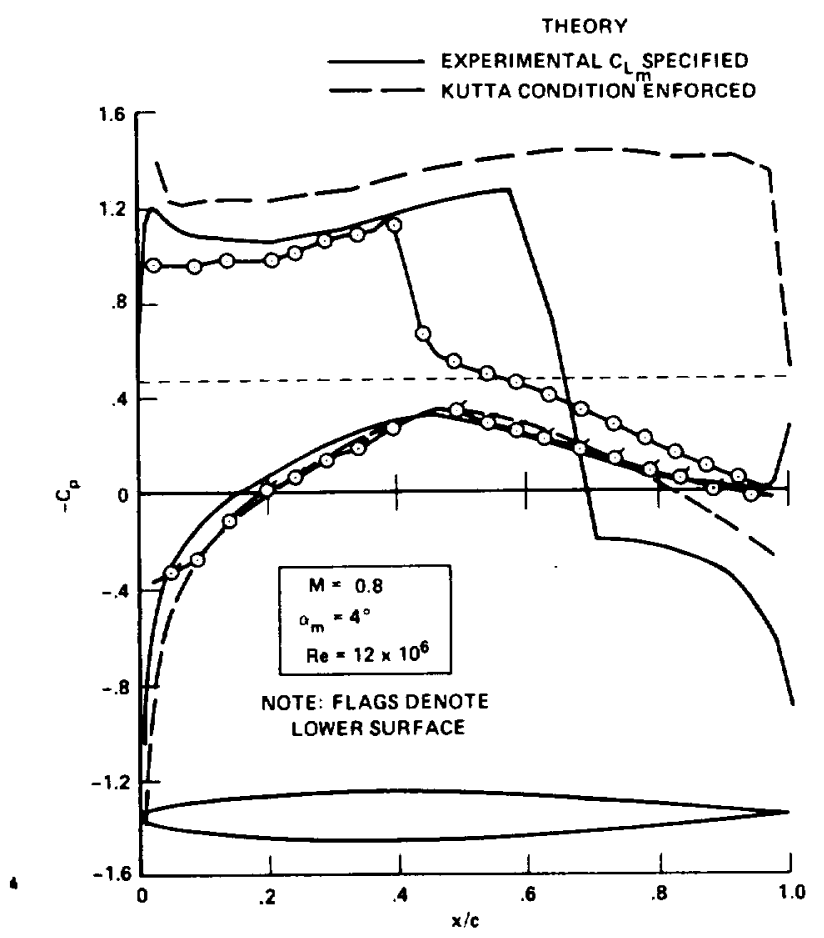

Fig. 11 Static pressure distribution compared with numerical solution of transonic small-disturbance equation.

show an extensive separation region at these conditions. The importance of viscous interactions, even in the steady flow, is shown in Fig. 11 where steady transonic small-disturbance theory (code TSFOIL) is compared with the experimental data. An attempt was made to force better agreement by matching the theoretical lift to the experimental lift, but this attempt was unsuccessful.

The quasisteady response for \pm 2 deg of pitch at a mean angle of attack of $4 \mathrm{deg}$ is shown in Fig. 12. As mentioned previously, the magnitude of the unsteady response is proportional to the distance between the limiting angles. Starting with the lower surface, the response decreases with distance from the trailing edge, vanishes at about midchord,

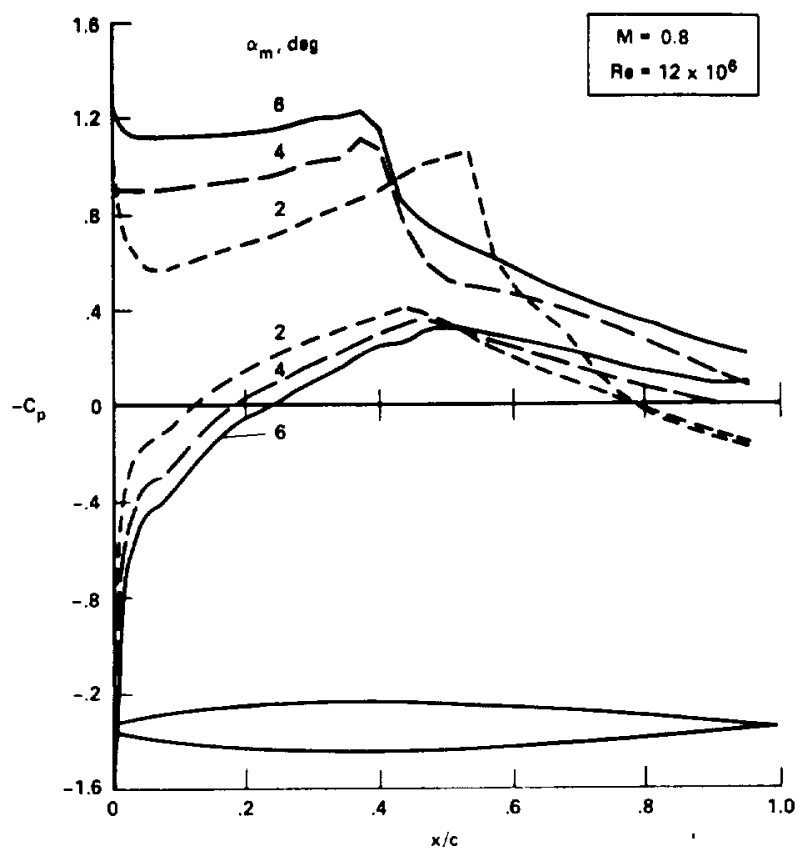

Fig. 12 Effect of mean angle on static pressure distribution for shock stall (condition 2).

and is out of phase with the motion over the forward half of the airfoil. (the phrase "out of phase" means decreasing suction with increasing angle of attack.) Turning to the upper surface and starting from the trailing edge, the magnitude decreases to zero at about $63 \%$ chord, is out of phase for about $20 \%$ of the chord, vanishes again at $43 \%$ chord, and increases toward the leading edge. On the upper surface there are two points of vanishing response $(X / C=0.43$ and 0.63$)$. This curious behavior is caused by the unnatural shock-wave movement. Usually a shock wave moves downstream with increasing angle of attack. In this case, shock stall causes the shock wave to move upstream with increasing angle of attack. This effect has been observed for a long time in steady-state testing (Ref. 27, for example), but the shock stall has an extremely important effect on the oscillating airfoil.

The unsteady pressures are presented in Fig. 13. The condition shown in Fig. 13, pitching \pm 1 deg at $25 \%$ chord, is a somewhat less exaggerated version of the \pm 2 deg excursions shown in the quasisteady response. At low frequencies the real part of the upper-surface response has both positive and negative lobes. The upper surface response is out of phase with the motion over the central portion of the airfoil. Both the theoretical and the experimental results shown in Figs. 6 and 8 indicate no cases in which the in-phase response has this particular shape. The authors are not aware of any unsteady aerodynamic theories that can predict this behavior-what is needed is a mathematical model that includes strong unsteady viscous interactions. With increasing frequency, the real part of the response curve changes shape and, at high frequencies, again resembles inviscid flow. The lower-surface pressure also changes somewhat with increasing frequency, but not as dramatically as that of the upper surface. The imaginary component is also quite different from that of the previous case. A large bump first appears on the upper surface at moderate frequencies and persists to high frequencies. The lower-surface response is much less affected by increasing frequency.

The predicted unsteady pressure distribution from linear subsonic theory is independent of mean angle of attack and is identical to that shown in Fig. 6; the linear theory is obviously inapplicable.

The aerodynamic transfer function has been computed and is shown in Fig. 14. The experimental data are compared with 


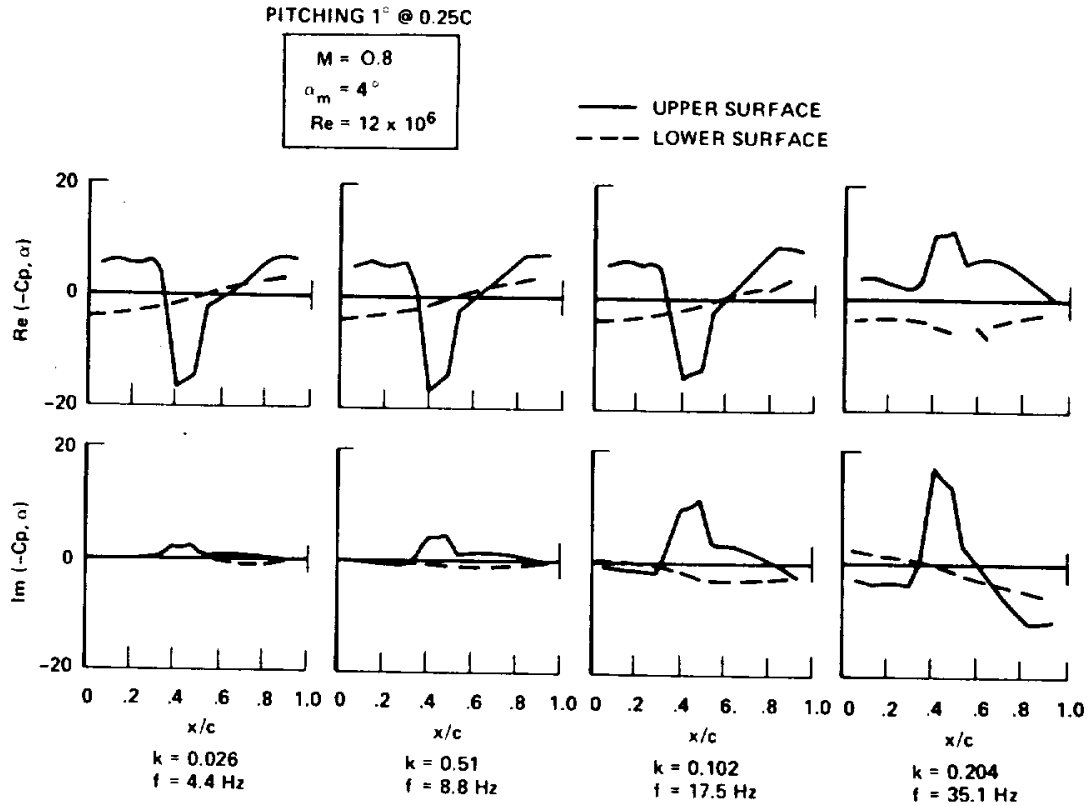

Fig. 13 Effect of frequency on unsteady pressure distributions.
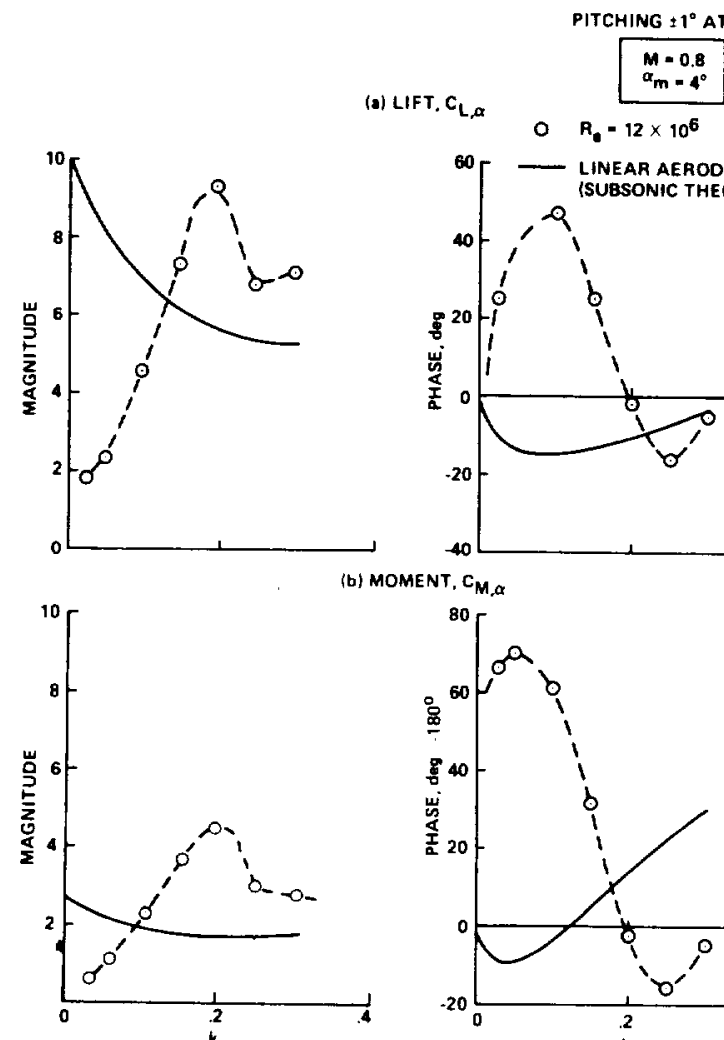

MOMENT, $C_{M, a}$
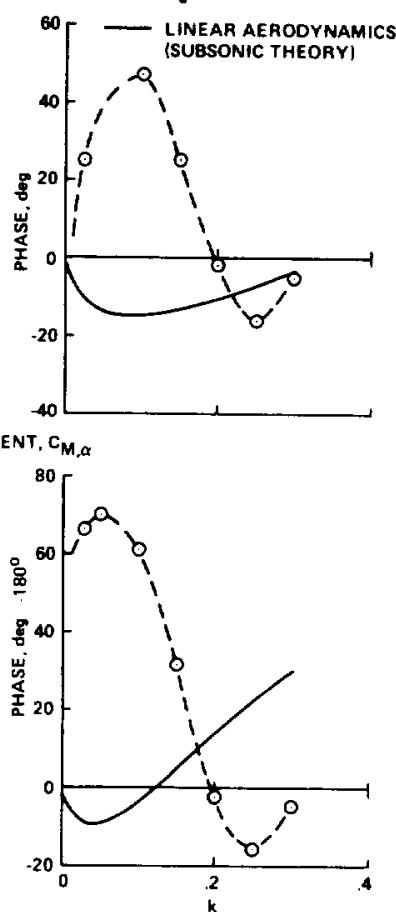

Fig. 14 Aerodynamic transfer function for shock-stall condition: a) lift coefficient, b) leading-edge moment coefficient (both per radian).

linear subsonic theory. Unlike Fig. 9, even the overall trends of the response are not well predicted by the linear theory. The magnitude of the loads peaks at about $k=0.2$ and is accompanied by a rapid phase shift that is somewhat reminiscent of a one degree-of-freedom resonance.

\section{Linearity and Modal Superposition}

The principle of superposition is the foundation on which the theory of aeroelasticity is built. The underlying assumption that the generalized forces are linear functions of the generalized coordinates leads to the concept of superimposing two modes of motion to predict a third unknown mode.

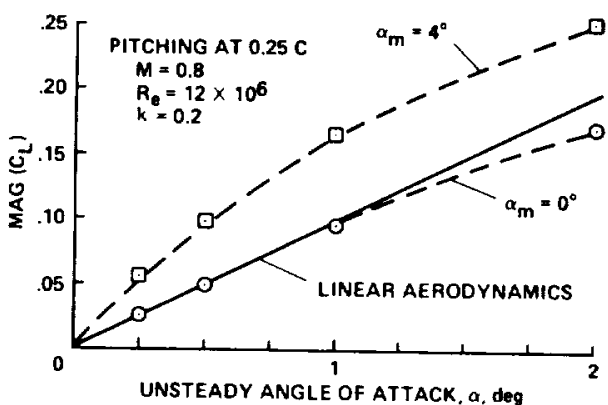

Fig. 15 Variation of unsteady lift coefficient magnitude with oscillatory angle of attack.

The principle of superposition was tested by first measuring the loads for three modes, assuming linearity, and finally combining two of the measured modes to predict the third. The two superimposed modes were pitching at $50 \%$ chord and plunging. The predicted mode (also measured) was pitching at $25 \%$ chord. The results are shown in Table 1. The unseparated flow tabulations show an error of less than $2 \%$ in magnitude and $2 \mathrm{deg}$ in phase over the entire frequency range. This error is about the level of experimental accuracy, and may be taken as a good indication of the validity of modal superposition. The tabulation for shock-induced separation at two Reynolds numbers shows errors in predicted and measured loads that are greater than can be accounted for by experimental techniques alone. It is known that flutter boundaries, for example, can be very sensitive to small changes in the phase of the loading with respect to the motion. The data show that the predicted lift coefficient slightly leads the motion while the measured lift coefficient slightly lags the motion. It can be concluded that the superposition principle is violated in this case, but the question of whether or not superposition can be assumed for practical calculations must await definitive aeroelastic computations.

The linearity of the dynamic loads was tested by varying the amplitude of the motion while keeping all the other parameters fixed. A representative example is presented in Fig. 15 for the magnitude of the lift coefficient. The attached flow, represented by $\alpha_{m}=0 \mathrm{deg}$, follows linear theory closely, deviating only slightly at the higher oscillatory angles of attack. The separated flow, $\alpha_{m}=4 \mathrm{deg}$, neither follows linear theory nor remains linear over an appreciable range of oscillatory angles of attack. This figure shows in another way 
Table 1 Measured and predicted unsteady lift coefficient

(NACA 64A010, $M=0.8$, pitching at $25 \%$ chord)

\begin{tabular}{|c|c|c|c|c|c|c|c|}
\hline \multirow[b]{2}{*}{$k$} & \multicolumn{3}{|c|}{ Measured } & \multicolumn{2}{|c|}{ Predicted } & \multicolumn{2}{|c|}{ Error } \\
\hline & $\operatorname{Re} \times 10^{-6}$ & $\mathrm{Mag}$ & $\mathrm{Ph}, \mathrm{deg}$ & Mag & Ph, deg & $\mathrm{Mag}, \%$ & $\Delta \mathrm{Ph}$, deg \\
\hline \multicolumn{8}{|c|}{ Unseparated flow, $\alpha_{m}=0$ deg } \\
\hline 0.025 & 12.6 & 0.171 & -8.8 & 0.169 & -9.0 & 1.1 & 0.2 \\
\hline 0.05 & 12.6 & 0.160 & -16.1 & 0.159 & -16.1 & 0.6 & 0.0 \\
\hline 0.10 & 12.6 & 0.134 & -26.7 & 0.133 & -28.3 & 1.0 & 1.6 \\
\hline 0.20 & 12.6 & 0.097 & -27.2 & 0.095 & -28.9 & 1.9 & 1.7 \\
\hline 0.20 & 6.7 & 0.091 & -28.2 & 0.092 & -29.5 & -0.7 & 1.3 \\
\hline \multicolumn{8}{|c|}{ Shock induced separation, $\alpha_{m}=4 \mathrm{deg}$} \\
\hline 0.20 & 11.9 & 0.166 & -0.9 & 0.153 & 4.6 & 7.8 & -5.5 \\
\hline 0.20 & 6.2 & 0.126 & $-\cdot 4.1$ & 0.132 & 3.3 & -5.4 & -7.4 \\
\hline
\end{tabular}

Linear superposition of measured pitching at $50 \%$ chord + measured plunging modes.

that separated flows are not necessarily superimposable. The deviations from linearity that are acceptable must again be found from practical computations and sensitivity analyses.

\section{Conclusions}

The data presented in this paper showed that transonic shock-wave/boundary-layer interactions have an important effect on unsteady pressure distributions and loads. For weak interactions, numerical computations are feasible and some progress has already been made in this direction. When the shock wave is strong enough to induce separation in the steady flow, the unsteady pressure distributions are strongly affected by the separation. Numerical modeling of steadystate separated flows is still not resolved, and the prediction of viscous, unsteady, transonic flows must await future developments.

The question of modal superposition and linearity has also been investigated experimentally. The superposition principle has been verified for unseparated flows, but some deviations were measured for separated flows. Setting the limits to which these principles can be relaxed for practical transonic applications must await detailed sensitivity calculations with realistic geometrical, elastic, and inertial parameters.

\section{References}

'Erickson, A. L. and Stephenson, J. D., “A Suggested Method of Analyzing for Transonic Flutter of Control Surfaces," NACA RM A7F30, Dec. 1947.

${ }^{2}$ Erickson, A. L. and Robinson, R. C., "Some Preliminary Results in the Determination of Aerodynamic Derivatives of Control Surfaces in the Transonic Speed Range by Means of a Flush-Type Electrical Pressure Cell," NACA RM A8H03, Oct. 1948.

${ }^{3}$ Sorenson, R. L., Wyss, J. A., and Kyle, J. C., "Preliminary Investigation of the Pressure Fluctuations in the Wakes of TwoDimensional Wings at Low Angles of Attack," NACA RM AS1G10, Oct. 1951.

A Wyss, J. A., and Sorenson, R. M., "An Investigation of the Control Surface Flutter Derivatives of an NACA $65,-213$ Airfoil in the Ames 16-Foot High Speed Wind Tunnel," NACA RM A51J10, Dec. 1951 .

${ }^{5}$ Wyss, J. A. and Montfort, J. C., "Effects of Airfoil Profile on the Two-Dimensional Flutter Derivatives for Wings Oscillating in Pitch at High Subsonic Speeds," NACA RM A54C24, May 1954.

${ }^{6}$ Wyss, J. A. and Herrera, R., "Effects of Angle of Attack and Airfoil Profile on the Two-Dimensional Flutter Derivatives for Airfoils Oscillating in Pitch at High Subsonic Speeds," NACA RM As4H12, Oct. 1954.

${ }^{7} \mathrm{Coe}$, C. F., "A Study of Local-Pressure Fluctuations Relative to Static-Pressure Distributions on Two-Dimensional Airfoils at High Subsonic Mach Numbers," NACA RM A55J11, Dec. 1955.

'Runyan, H. L., Woolston, D. S., and Rainey, A. G., "Theoretical and Experimental Investigations of the Effects of Tunnel Walis on the Forces of an Oscillating Airfoil in TwoDimensional Subsonic Compressible Flow," NACA Rept. 1262, 1956.

${ }^{9}$ Clevenson, S. and Widmayer, E., Jr., "Experimental Measurements of Forces and Moments on a Two-Dimensional Oscillating Wing at Subsonic Speeds," NACA TN 3686, June 1956.
${ }^{10}$ Rainey, A. G., "Measurement of Aerodynamic Forces for Various Mean Angles of Attack on an Airfoil Oscillating in Pitch and on Two Finite-Span Wings Oscillating in Bending with Emphasis on Damping in the Stall," NACA Rept. 1305, 1957.

"Bratt, J. B., Raymer, W. G., and Townsend, J.E.G., "Measurements of the Direct Pitching-Moment Derivatives for TwoDimensional Flow at Subsonic and Supersonic Speeds and for a Wing of Aspect Ratio 4 at Subsonic Speeds," R\&M 3257, British A.R.C., Jan. 1959.

${ }^{12}$ Acum, W.E.A., "The Comparison of Theory and Experiment for Oscillating Wings," AGARD Manual on Aeroelasticity, 1968 Revision, Part II, 1962, Chap. 10 (also released as N.P.L. Aeronautical Rept. 1005, 1962).

${ }^{13}$ Tijdeman, H., "On the Motion of Shock Waves on an Airfoil with Oscillating Flap in Two-Dimensional Transonic Flow," NLR TR 75038 U, NLR, Amsterdam, The Netherlands, March 1975.

${ }^{14}$ Tijdeman, H., Schippers, P., and Persoon, A. J., "Unsteady Airloads on an Oscillating Supercritical Airfoil," Paper presented at AGARD SMP Specialist's Meeting. Unsteady Airloads in Separated and Transonic Flow, Lisbon, Portugal, April 18, Oct. 1977.

${ }^{15}$ Tijdeman, H., "Investigations of the Transonic Flow Around Oscillating Airfoils," NLR TR 77090 U, NLR, Amsterdam, The Netherlands, 1977.

${ }_{16}$ Proceedings of AGARD FDP Symposium on Unsteady Aerodynamics, Ottawa, Canada, Sept. 26-28, 1977, AGARD-CP-227, 1978.

${ }^{17}$ Proceedings of AIAA Dynamics Specialist Conference, San Diego, Calif., March 24-25, 1977, AIAA, New York, 1977.

${ }^{18}$ Malcolm, G. H. and Davis, S. S., "New NASA-Ames Wind Tunnel Techniques for Studying Airplane Spin and Two-Dimensional Unsteady Aerodynamics," AGARD CP-235, 1978, pp. 3-1-3-12; (also in NASA CP-2045, 1979, pp. 671-688).

${ }_{19}$ Davis, S. S. and Malcolm, G. N., "Experiments in Unsteady Transonic Flow," AlAA Paper 79-0769, AIAA/ASME/ASCE/AHS 20th SDM Conf., St. Louis, Mo., April 1979.

${ }^{20}$ Davis, S. S., "Computer/Experiment Integration for Unsteady Aerodynamic Research," ICIASF " 79 Record, International Congress on Instrumentation in Aerospace Simulation Facilities, Monterey, Calif., Sept. 24-26, 1979.

${ }^{21}$ Davis, S. and Malcolm, G., “Experimental Unsteady Aerodynamics of Conventional and Supercritical Airfoils," NASA TM 81221, Aug. 1980.

${ }^{22}$ Olsen, J. J., "AGARD Standard Configurations for Aeroelastic Applications of Transonic Unsteady Aerodynamics, Part III, Candidate Airfoil Data (+Addendum)," AFFDL-TM-78-6-FBR, Part III, Jan. 1978.

${ }^{23}$ Bland, S. R., "AGARD Two-Dimensional Aeroelastic Configurations," AGARD-AR-156, Aug. 1979.

${ }^{24}$ Magnus, R. J., "Calculations of Some Unsteady Transonic Flows About the NACA 64A006 and NACA 64A010 Airfoils," AFFDL-TR-77-46, July 1977.

${ }^{29}$ Stahara, S. S., "Operational Manual for Two-Dimensional Transonic Code TSFOIL," NASA CR-3064, Dec. 1978.

${ }^{26} \mathrm{Chyu}, \mathrm{W}$. and Davis, S., "Calculation of Unsteady Transonic Flow over an Arbitrary Airfoil," AIAA Paper 79-1554, AIAA 12th Fluid and Plasma Dynamics Conf., Williamsburg, Va., July 1979.

${ }^{27}$ Johnson, D. A. and Bachalo, W. D., "Transonic Flow About a Two-Dimensional Airfoil-Inviscid and Turbulent Flow Properties," AIAA Journal, Vol. 18, No. I, Jan. 1980, pp. 16-24. 